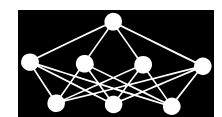

\title{
A NEW METHOD FOR NUCLEAR ACCIDENT SOURCE TERM INVERSION BASED ON GA-BPNN ALGORITHM
}

\author{
Y. Ling, C. Chai, W. Hou
}

\begin{abstract}
Rapid and accurate prediction and evaluation of accident consequences can provide scientific basis for decision-making of nuclear emergency measures. Accident source term estimation under reactor accident conditions is an important part of nuclear accident consequence evaluation. In order to accurately estimate the information of radioactive source terms released from nuclear power plants to the environment, an inversion model of accident source terms based on BP neural network algorithm (BPNN) was constructed. And to resolve the defect that BPNN is easy to fall into local minimum during training process, genetic algorithm (GA) was used to optimize the weights and thresholds of BPNN. In this paper, referring to the release rates of radioactive source term from the Fukushima nuclear accident. The release rates of ${ }^{131} \mathrm{I}$ and ${ }^{137} \mathrm{Cs}$ diffused into the environment in stable atmosphere were taken as the two target outputs of the GA-BPNN, and the meteorological data for one hour at fixed monitoring points were taken as the target inputs. And the simulation results showed that for the release rate of ${ }^{131} \mathrm{I}$ and ${ }^{137} \mathrm{Cs}$, the mean relative errors of the training and the testing sample sets were both below $2 \%$ which indicates that the GA-BPNN model not only improves the shortcoming of BPNN, but also increases the speed and accuracy of source term inversion.
\end{abstract}

Key words: nuclear accident, source term, inversion, genetic algorithm, back propagation neural network

Received: 2017-01-12

DOI: $10.14311 / \mathrm{NNW} .2019 .29 .006$

Revised and accepted: 2019-04-24

\section{Introduction}

Three severe nuclear accidents in human history have shown the risk of leakage of radioactive material for nuclear power plants, and some appropriate measures play an important role in making recommendations for the protection of the public and

\footnotetext{
*YongSheng Ling - corresponding author, Qing Shan, WenBao Jia; Department of Nuclear Science and Engineering, Nanjing University of Aeronautics and Astronautics, Nanjing 211106, China; Collaborative Innovation Center of Radiation Medicine of Jiangsu Higher Education Institutions, Suzhou 215021, China; E-mail: lingyongsheng@nuaa.edu.cn

${ }^{\dagger}$ ChaoJun Chai, Wenyu Hou, DaQian Hei; Department of Nuclear Science and Engineering, Nanjing University of Aeronautics and Astronautics, Nanjing 211106, China.
} 
environment. Reliable source term estimation method can provide the necessary data for the evaluation of nuclear accident consequences and decision making. Previous experience showed that when the serious nuclear accident happened, reactor core damaged inside the equipment severely, thus the data were often difficult to be obtained. Therefore, a viable approach to access the source term is to use some kind of inverse modeling based on atmospheric transport modeling and environmental monitoring data, which is called nuclear accident source term inversion [1].

The source term studied in this paper refers to radioactive substances released from specific sources into the atmosphere, including the release of radionuclides species, quantity, release rate and mode [2]. At present, inversion models available for accident source term inversion are least square estimation [3], Genetic Algorithm (GA) [4], Kalman Filter and Extended Kalman Filter [5], Artificial Neural Network (ANNs) [6]. Jeong H.J. et al. (2005) estimated the source release rate of Yenong-Kwang nuclear power station using the least square estimation method combined with the Gauss plume atmospheric diffusion model and meteorological data [3]. This method is more suitable for studying linear systems. The GA is easy to combine with other diffusion models, but it depends on the initial population selection [4]. Sun S D et al. (2019) used air dispersion models and environmental measurements to determine the atmospheric release rate with EnKF [5]. The leakage and diffusion of radioactive materials in a nuclear accident is complex and non-linear process.

The first three methods rely on the position of the measurements and the air dispersion scenario strongly. Back propagation neural network (BPNN) algorithm has the sharp advantages of high fault tolerance, self-organization, adaptive and self-learning [6]. This method is more suitable for multivariate nonlinear systems, which can maximize the probability of distilling the source information corresponding to system variables from large amounts of independent information and has good explanation ability. However, an inherent defect that led to fall into local minimum trap during the BPNN learning process. To overcome the problem and improve convergence speed, GA is usually used to optimize the BPNN for enhancing the estimation accuracy. Xue X H et al. (2015) constructed the hybrid model based on the combination of GA and BPNN for the purpose of improving the safety monitoring accuracy and stability of high slopes [4]. The learning process of the BPNN was optimized by using the GA to obtain the optimal solution of network connection weights in order to improve nonlinear magneto telluric (MT) inversion by Wang et al. (2018) [7].

In the nuclear accident consequence assessment, the radionuclide with high yield, medium half-life, more obvious radiation biological effect, gaseous or volatile eventually released to the environment need to be considered [8]. Inhaling or ingesting ${ }^{137} \mathrm{Cs}$ distributes the radioactive material in the soft tissues, especially the muscles, and increases the risk of cancer [9]. The equivalent ${ }^{131} \mathrm{I}$ release is used as a criterion for serious accidents in the international nuclear incident classification table. It is difficult to get the proportion of each radioactive substance when many kinds of radioactive substances diffuse into the atmosphere. In this paper, the method based on GA-BPNN was proposed for nuclear accident source term inversion by the ability of BPNN self-learning, which took the release rates of ${ }^{131} \mathrm{I}$ and ${ }^{137} \mathrm{Cs}$ as the two output targets. The inputs of neural network were composed 
of the environmental monitoring data of nuclear accidents and gamma radiation dose rate of nuclear power plants. The release rates obtained in this study are the rates of stable diffusion of radionuclides in the atmosphere after a period of time. The accuracy of the inversion model was analyzed by comparing the actual output with the expected output. The experimental results showed that the GA-BPNN could make full use of the global searching capability of the GA and the local optimization of the BPNN. To a certain extent, the operation efficiency and calculation accuracy of the genetic neural network were improved Compared with the single BPNN inversion model.

\section{Methodology}

\subsection{BP neural network}

Artificial neural network is suitable for modeling multivariate nonlinear complex system relationships and has the advantages of small error and high precision. The most commonly used is the neural network based on error back propagation multilayer feedforward neural network algorithm (Multiple-layer feed forward network), called BP neural network. BP neural network model is composed of input layer, output layer and hidden layer. The learning process of BP neural network consists of forward propagation and backward propagation [10]. A typical three layers BP neural network was shown in the Fig. 1, where the input signal, output of hidden layer, output of output layer, the target signals are represented by $X_{i}, Y_{h}, Z_{j}$ and $T_{j}$ respectively. The connection weight from the input node " $i$ " to the node " $h$ " of hidden layer is represented by $\omega_{i h}$, and the connection weight from the node of hidden layer $h$ to the node of the output layer $j$ is represented by $\omega_{h j} . N_{1}, N_{2}$, $N_{3}$ respectively represent the numbers of the input layer, hidden layer, and output layer node.

The basic idea of BP learning algorithm is to minimize the back propagation of error by adjusting and modifying the connection weights $\omega$ of the network until the error meets the conditions. The essence of BP neural network is to find a mapping

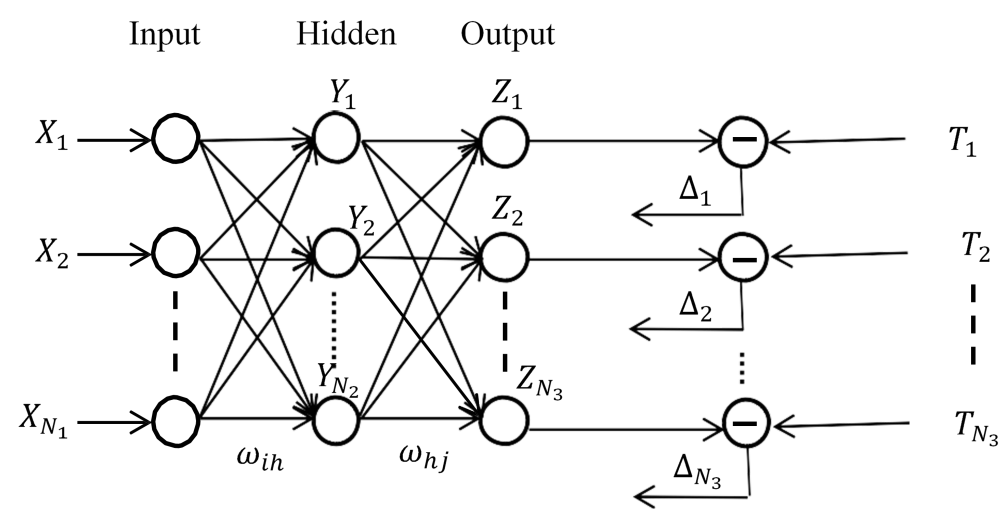

Fig. 1 Schematic diagram of a typical BP neural network structure. 
function from input variables to output variables, and the mathematical theory has proved that it can achieve an arbitrary nonlinear mapping process. It needs to be pointed out that once the BP neural network has been trained well, only the forward propagation instead of back propagation needs to be used. So, the source inversion method based on BP neural network greatly saves more time than other inversion methods, which is suitable for nuclear accident source term inversion.

\subsection{Genetic algorithm}

The BP training may lead to a local rather than a global error minimum. The local error minimum that has been found may be satisfactory, but if it is not, a network with more neurons may do better job. Alternatively, one might run the problem using several different sets of initial conditions to see if they lead to the same or different solutions. Further, in the present work, care has been taken to avoid localization by properly determining the number of hidden neurons. Although this number is chosen on trial and error basis, trials started with the average value of number of inputs and outputs and then varied around that value to find the best number of hidden neurons. Another problem with BP is its slow converging nature that leads to little longer training time.

GA is a theory and Mendel's genetic theory proposed a parallel random search optimization algorithm based on the simulation of natural genetic mechanism and biological evolution theory of Darwin's evolution [11]. The basic idea is that the nature biological evolution principle of "natural selection and survival of the fittest" is introduced to optimize the parameters of the structure of population coding, according to the choice of adaptation degree function and through genetic selection, crossover and mutation screening of individual, adaptation of a body is preserved, adaptation of individuals are eliminated, new groups not only inherits a generation of information and better than generation. Such repeat cycles until meet the condition [12].

The basic elements of GA include chromosome coding, fitness function, genetic operation and operation parameters. The chromosome coding method is the coding method of the training sample.

\subsection{Optimization by GA}

GA with probability choice as the main means, adept at global search, the organic combination of the two algorithms, enhance the learning ability of the network, many scholars did a lot of research work management on how to combine genetic algorithm and BP neural network integration [4].

Standard GA flow was adopted to simultaneously optimize BPNN weights and bias for improving the prediction accuracy in this study. Optimization by GA could shorten the training time and enhanced the convergence speed with determining suitable initial threshold of each node and connection weights between nodes instead of beginning from a random number. The process of BPNN optimized by GA included group initialization, fitness function, selection operation, crossover operation and mutation operation. In the process, fitness was regarded as a stopping criterion. The optimization procedure was planned in detail as shown in Fig. 2. 
Ling Y. et al.: A new method for nuclear accident source term...

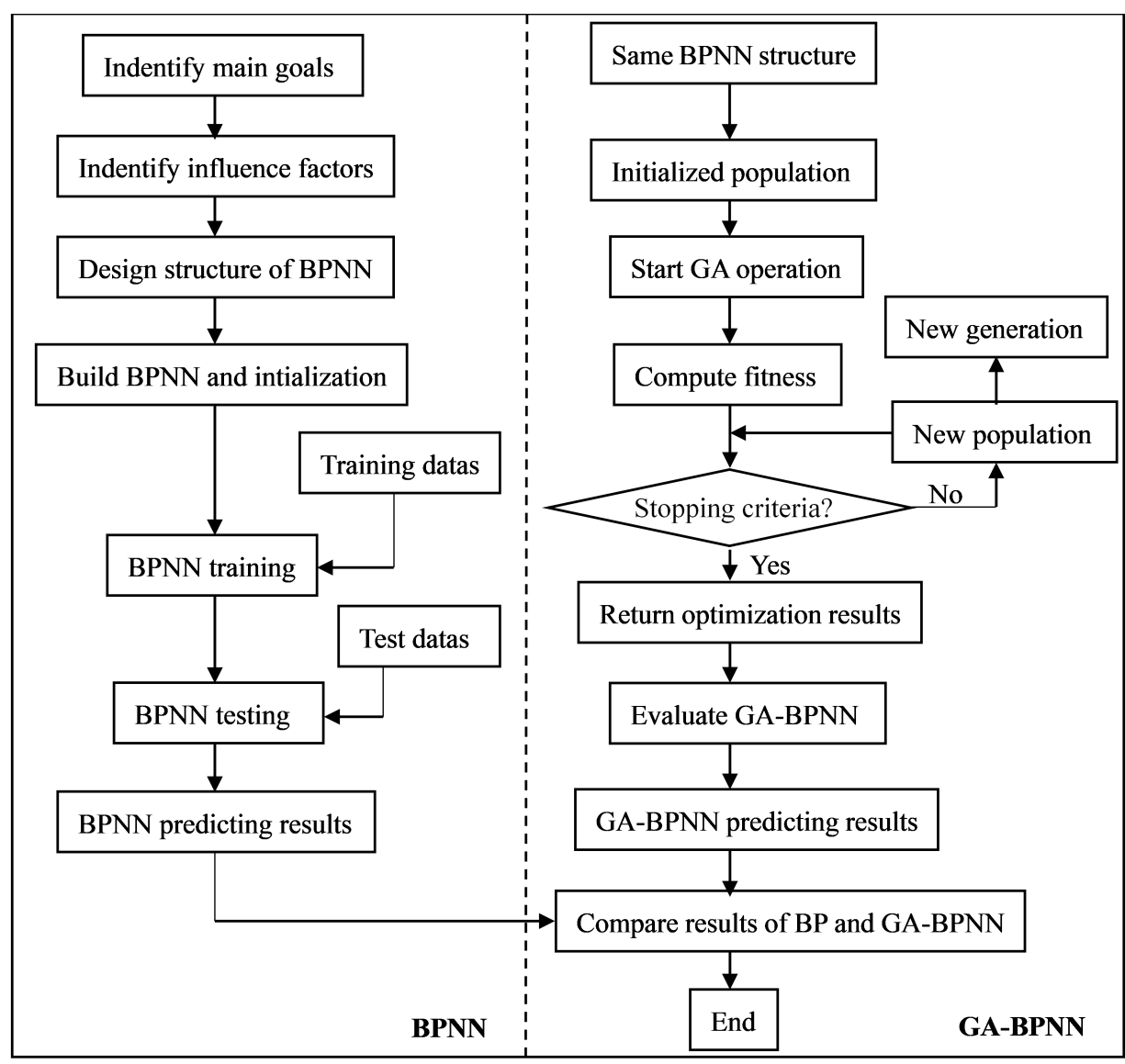

Fig. 2 Flow chart of the GA-BP neural network algorithm in the inversion of the source term.

\section{Numerical experiment}

\subsection{Design of BPNN structure}

According to the above-mentioned analysis, 7 of input layer nodes (wind direction, wind speed, atmospheric stability, precipitation type, gamma radiation dose rate, location of the measurement, release height) and 2 of output layer nodes (release rate of ${ }^{131} \mathrm{I}$ and ${ }^{137} \mathrm{Cs}$ ) were determined in BPNN.

The number of hidden layer nodes was determined by the numerical experiments performed with MATLAB R2016a (see Section 4.1)

\subsection{Data collection}

Reference to the release rate range of the Fukushima nuclear accident and the release type PWR1-PWR9 of accident in the reactor safety study of the United States [13], the release rate of ${ }^{131} \mathrm{I}$ was chosen to be between $10^{11}$ and $10^{14} \mathrm{~Bq} \cdot \mathrm{h}^{-1}$, 
and the release rate of ${ }^{137} \mathrm{Cs}$ was chosen to be between $10^{12}$ and $10^{15} B q \cdot \mathrm{h}^{-1}$. The expected outputs of BPNN were taken in the above ranges. In the ST-DOSE module of InterRAS, the environmental impact factors such as wind direction, wind speed, wind distance, atmospheric stability and precipitation type were set up, and the gamma dose rates monitored within one hour from the position of $1.0 \mathrm{~km}, 2.0 \mathrm{~km}, 5.0 \mathrm{~km}, 25.0 \mathrm{~km}$ and $50.0 \mathrm{~km}$ to the nuclear power plant after the nuclear accident occurs were obtained [14]. Atmospheric stability has six levels, A, $\mathrm{B}, \mathrm{C}, \mathrm{D}, \mathrm{E}$ and $\mathrm{F}$ which is replaced by $1,2,3,4,5$ and 6 for the convenience of later data processing. (The experimental background of this study was the stable release of radionuclides in the atmosphere, that is, only considering the atmospheric stability of D). Precipitation could be divided into NONE, Light rain, Moderate rain, Heavy rain, Light snow, Moderate snow and Heavy snow, which were also replaced by $1,2,3,4,5,6$ and 7 . The experimental data contained 20000 sets of training data and 1000 sets of test data, and some of the data was shown in Tab. I.

\subsection{MATLAB}

All of numerical experiments were performed by coding with neural network toolbox in MATLAB R2016a. The training function 'trainlm', transfer function 'tansig' in the neural network were used. The training time, training target error and study rate was $100,0.00001$ and 0.01 , respectively.

\section{Results and discussion}

\subsection{Determination of the number of hidden layer nodes}

A typical three-layer BPNN was established with 7 input layer nodes, 2 output layer nodes and a hidden layer. If the number of hidden layer nodes are too few, the neural network will not be trained. However, if the number of hidden layer nodes are too many, it may prolong the network training time, and it is easy to fall into the local extreme points, and the "over fitting" phenomenon appears. To determine the number of hidden layer nodes, trainings were performed by MATLAB at the number of hidden layer nodes that changed from 20 to 70 . The training results were shown in Fig. 3 and Fig. 4. From Fig. 3 and Fig. 4, the training means square errors and the training errors decreased with the increased nodes and appeared unstable. Low error at a node number of 65 indicated that the prediction ability of model was stronger. Therefore, a 7-65-2 BPNN was determined since more nodes mean longer training time, which contained 7 input layer nodes, 65 hidden layer nodes and 2 output layer nodes.

\subsection{Inversion by BPNN and optimization by GA}

The GA optimization was performed on the above 7-65-2 BPNN. The population size, evolution algebra, probability of crossover and mutation probability were 200 , $0.2,0.2$ and 0.1 , respectively. The fitness curve and error curve of the genetic algorithm were shown in Fig. 5. The red curve represented the optimal individual 
Ling Y. et al.: A new method for nuclear accident source term...

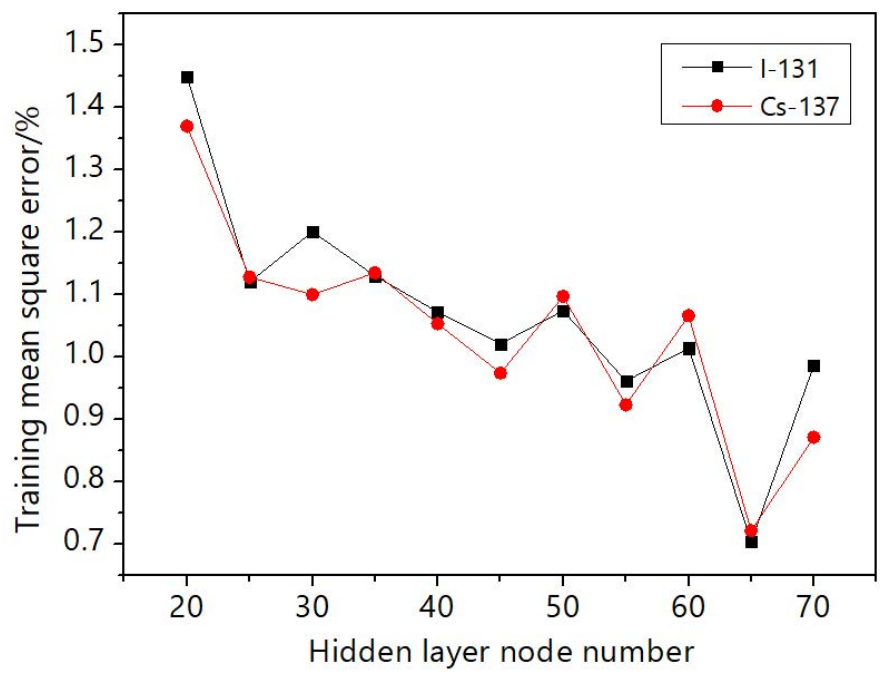

Fig. 3 Training mean square errors at different numbers of hidden layer nodes.

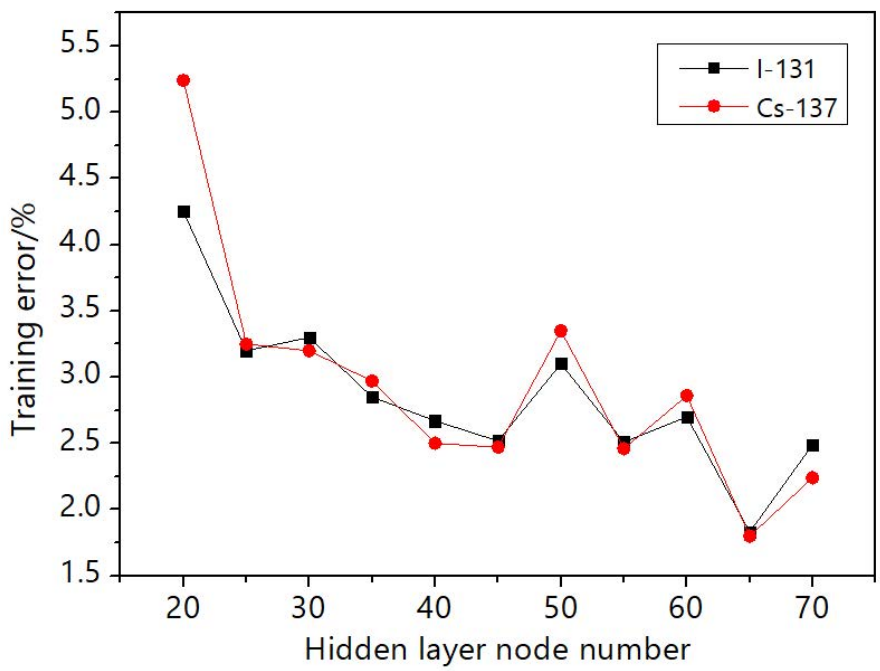

Fig. 4 Training errors at different numbers of hidden layer nodes.

variation for ${ }^{131} \mathrm{I}$ in the process of genetic algorithm optimization and the blue represented the optimal individual variation for ${ }^{137} \mathrm{Cs}$.

Fitness value was used to evaluate the superiority of the individual. A bigger fitness value suggested a better individual that had more chances to reproduce, whose excellent properties could be inherited. From Fig. 5, the fitness curve appeared smooth above 60 of generation, and it indicated that the weights and thresholds have been optimized. 
Neural Network World 2/2019, 71-82
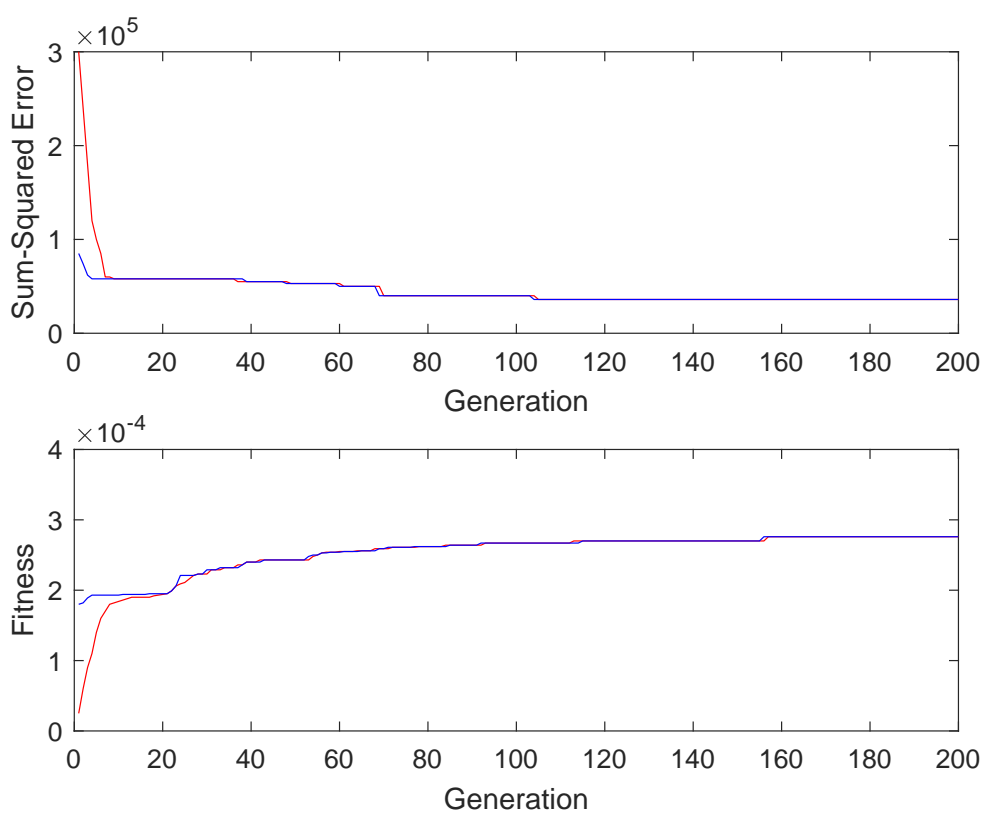

Fig. 5 The fitness curve and error curve.

Thus, the weights and thresholds optimized were used to optimize the original BPNN. In principle, BPNN uses input data to achieve inversion target through testing process. 90 groups were randomly selected to compare the prediction results with the actual targets from the 1000 sets of test samples. In Fig. 6 , about $67 \%$ of the test samples of ${ }^{131} \mathrm{I}$ and ${ }^{137} \mathrm{Cs}$ can be achieved the desired output after BPNN inversion. The error of BPNN was large, so the performance of the model needs

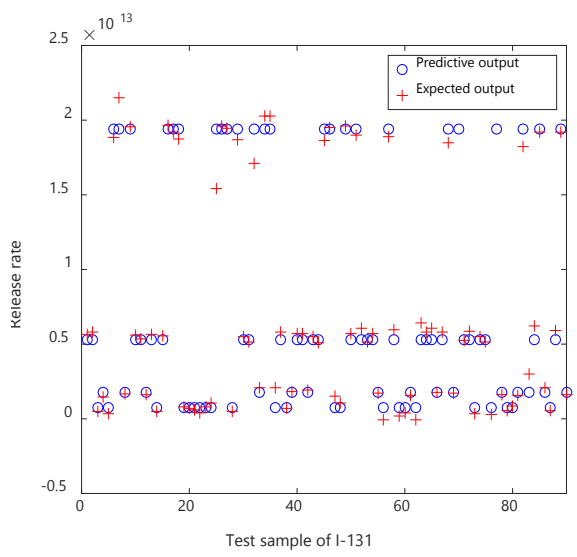

(a) Predicted result of ${ }^{131} \mathrm{I}$ by BPNN.

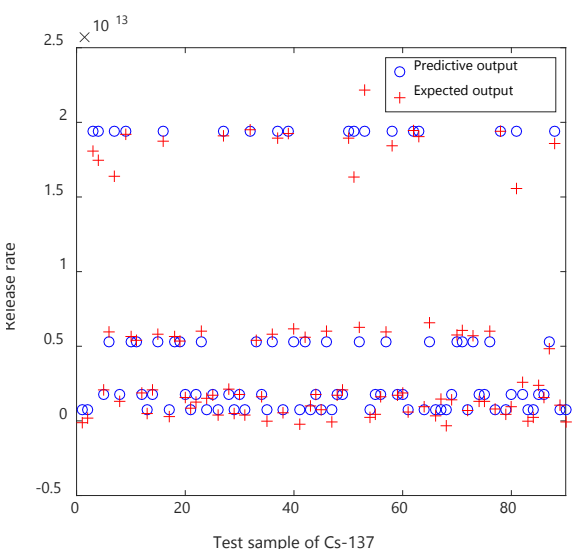

(b) Predicted result of ${ }^{137} \mathrm{Cs}$ by BPNN.

Fig. 6 The predicted results of BPNN. 
to be further improved. After optimization, the inversion result of the GA-BPNN model was shown in Fig. 7. From Fig. 7, more than $90 \%$ of test relative training errors for ${ }^{131} \mathrm{I}$ or ${ }^{137} \mathrm{Cs}$ by GA were below 0.2 . This indicated that the predicted data obtained by GA-BPNN were much closer to the source release rate of the nuclear power plant when serious accident occurred. It realized that the accuracy of source term inversion by BPNN could be improved after optimization by GA.

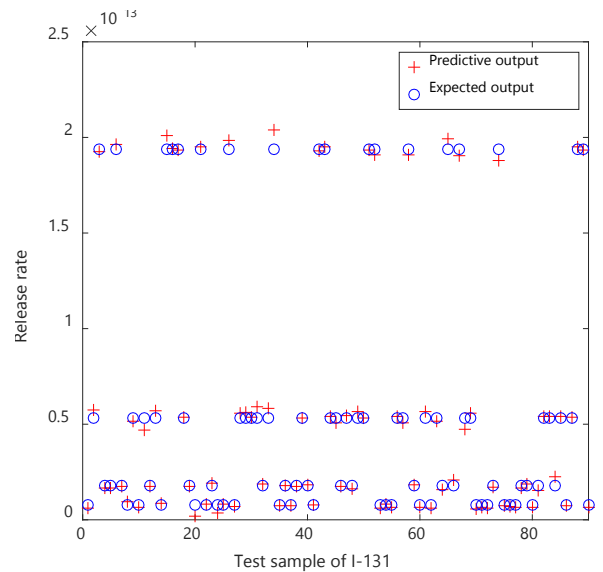

(a) Predicted result of ${ }^{131} \mathrm{I}$ by GA-BPNN

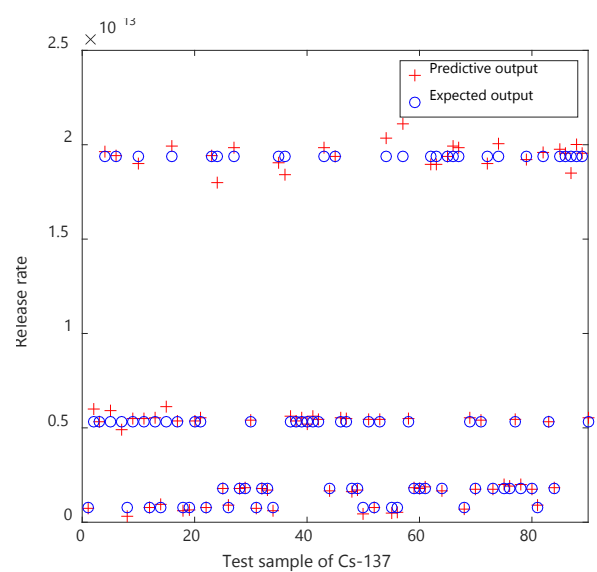

(b) Predicted result of ${ }^{137} \mathrm{Cs}$ by GA-BPNN.

Fig. 7 The predicted results of GA-BPNN.

Prediction time, mean square error and mean test relative error were selected to characterize model performance. From Tab. II, the model proved to be more accurate than conventional methods, with the mean training relative errors for ${ }^{131} \mathrm{I}$ and ${ }^{137} \mathrm{Cs}$ below $2 \%$. This indicated that the model can be used to inversed $98 \%$ of the source information based on the environmental data from monitoring station around the nuclear power plant and the doses of radionuclides. In addition, training error and training time of BPNN inversion model was obviously reduced, and the prediction stability was improved after optimization by GA. The mean test errors after optimization by GA were below 0.1 , and it decreased significantly relative to the errors by BPNN, especially for ${ }^{131}$ I. This method can provide source information quickly and accurately for nuclear accident emergency.

\section{Conclusion}

In this paper, a BPNN has been proposed for source term inversion during a nuclear accident, which contains 7 input layer nodes, 65 hidden layer nodes and 2 output layer nodes. The neuron network was also optimized by GA for overcoming the inherent defect that leads to fall into a local minimum trap during BP neural network learning process. The conclusions by numerical experiments performed with MATLAB software could be described as the following:

1. The mean training relative errors for ${ }^{131} \mathrm{I}$ and ${ }^{137} \mathrm{Cs}$ are both below $2 \%$. The mean test relative errors for ${ }^{131} \mathrm{I}$ and ${ }^{137} \mathrm{Cs}$ are both below $12 \%$. It indicated 


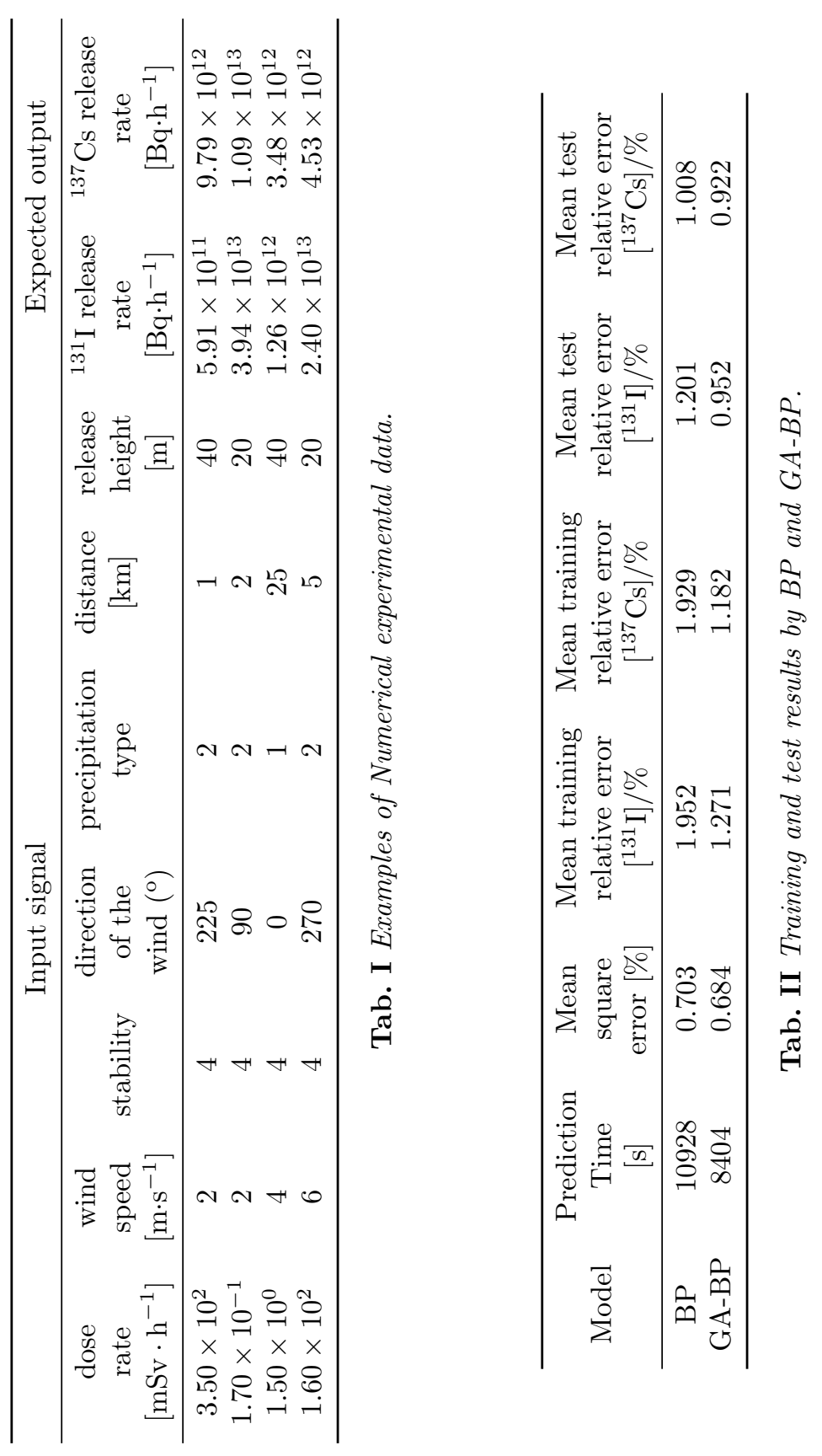


that the method refers to the 7-65-2 BPNN could be used for source term inversion during a nuclear accident once the BPNN were trained well by a lot of data available.

2. The mean training relative error, mean square error of training, training time and test relative error after optimization by GA are all less than those values by BP. It suggested that the optimization by GA could afford an enhancement in accuracy of source term inversion during a nuclear accident.

Although only the poor real data can be obtained since nobody can perform a series of appropriate experiments with nuclides, the method described in this paper can use the data from nuclear accident consequence system developed by each nuclear power plant to build the BPNN model in emergency preparedness, and estimates the source term with the real data during an accident.

\section{Acknowledgement}

This work was supported by the Fundamental Research Funds for the Central Universities (Grant No. NS2017037) and the National Natural Science Foundation of China (Grant No. 11405086). And this work was supported by a Project Funded by the Priority Academic Program Development of Jiangsu Higher Education Institutions (PAPD).

\section{References}

[1] NASSTROM J., SUGIYAMA G., et al. The National Atmospheric Release Advisory Center modelling and decision-support system for radiological and nuclear emergency preparedness and response. International Journal of Emergency Management, 2007, 4(3), pp. 524-550, doi: 10.1504/ijem.2007.014301.

[2] SHI Z. Nuclear and radiation emergency preparedness and response. Beijing: Atomic Energy Press, 2010, pp. 122-135.

[3] JEONG H., KIM E., et al. Determination of the source rate released into the environment from a nuclear power plant. Radiation Protection Dosimetry, 2005, 113(3), pp. 308-13, doi: $10.1093 / \mathrm{rpd} / \mathrm{nch} 460$.

[4] XUE X., LI Y., YANG X., CHEN X., XIANG J. Prediction of slope stability based on GABP hybrid algorithm. Neural network world, 2015, 25(2), pp. 189-202, doi: 10.14311/NNW. 2015.25.010.

[5] SUN S., LI X., LI H., SHI J., FANG S. Site-specific (Multi-scenario) validation of ensemble Kalman filter-based source inversion through multi-direction wind tunnel experiments. Journal of Environmental Radioactivity, 2019, 197, pp. 90-100, doi: 10.1016/j.jenvrad.2018. 12.005 .

[6] ASL B., SHARAFAT A., SETAREHDAN S. An adaptive backpropagation neural network for arrhythmia classification using R-R interval signal. Neural network world, 2012, 22(6), pp. 535-548, doi: 10.14311/NNW. 2012.22.033.

[7] WANG H., LIU M., et al. 2018. Magnetotelluric inversion based on BP neural network optimized by genetic algorithm. Chinese Journal of Geophysics, 2018, 61(4), pp. 1563-157, doi: $10.6038 /$ cjg2018L0064.

[8] KATATA G., TERADA H., NAGAi H., CHINO M. Numerical reconstruction of high dose rate zones due to the Fukushima Dai-ichi Nuclear Power Plant accident. Journal of Environmental Radioactivity, 2012,112, pp. 2-12, doi: 10.1016/j.jenvrad.2011.09.011. 


\section{Neural Network World 2/2019, 71-82}

[9] STOHL A., SEIBERT P., et al. Xenon-133 and caesium-137 releases into the atmosphere from the Fukushima Dai-ichi nuclear power plant: determination of the source term, atmospheric dispersion, and deposition. Atmospheric Chemistry and Physics, 2009, 12, pp. 2313-2343, doi: 10.5194/acpd-11-28319-2011.

[10] GALESHCHUK S. Neural networks performance in exchange rate predictio. Neurocomputing, 2016, 172, pp. 446-452, doi: 10.1016/j.neucom.2015.03.100.

[11] MOURET J. Evolutionary Adaptation in Natural and Artificial Systems. 2015, 19(6), pp $1606-1619$.

[12] ÖRKCÜ H., BAL H. Comparing performances of back propagation and genetic algorithms in the data classification. Expert Syst. Appl., 2011, 38, pp. 3703-3709, doi: 10.1016/j. eswa. 2010.09 .028

[13] HIROSE K. 2011 Fukushima Dai-ichi nuclear power plant accident: summary of regional radioactive deposition monitoring results. Journal of Environmental Radioactivity, 2012, 111, pp. 13-17, doi: 10.1016/j.jenvrad.2011.09.003.

[14] REGE M., TOCK R. A simple neural network for estimating emission rates of hydrogen sulfide and ammonia from single point sources. Air and Waste Manage, 1996, 46, pp. 953962, doi: 10.1080/10473289.1996.10467530. 